\title{
Solid Lipid Nanoparticles and Nanostructured Lipid Carriers of Loratadine for Topical Application: Physicochemical Stability and Drug Penetration through Rat Skin
}

\author{
Melike Üner ${ }^{1^{*}}$, Ecem Fatma Karaman ${ }^{1}$ and Zeynep Aydoğmuş ${ }^{2}$ \\ Istanbul University, Faculty of Pharmacy, ${ }^{1}$ Department of Pharmaceutical Technology, ${ }^{2}$ Department of Analytical Chemistry, \\ 34116 Beyazit, Istanbul, Turkey
}

*For correspondence: Email: melikeuner@yahoo.com; Tel: +90 212 4400000; Fax: +90 2124400252

\begin{abstract}
Purpose: To prepare solid lipid nanoparticles (SLN) and nanostructured lipid carriers (NLC) of loratadine (LRT) for the treatment of allergic skin reactions.

Methods: SLN and NLC were prepared by high pressure homogenization method. Their entrapment efficiency (EE) and loading capacity (LC) were determined. The physical stability of nanoparticles was investigated during 6 months of storage at room temperature (RT), 4 and $40^{\circ} \mathrm{C}$. Fourier transform infrared spectroscopy (FTIR), differential scanning calorimetry (DSC) and laser diffraction (LD) were used for the investigation of drug:excipient compatibility, thermal behaviour and particle size of the nanoparticles. In vitro release and ex vivo skin penetration of LRT were studied. Nanoemulsions (NE) were also prepared and characterized for comparison.

Results: Nanoparticles sizes ranged from $0.222 \pm 0.011 \mu \mathrm{m}$ to $0.252 \pm 0.014 \mu \mathrm{m}$ ( $D 50$ as a value based on the volume distribution, the maximum particle diameter below which $50 \%$ of the sample volume exists) They were obtained with high drug payloads (> 90.67\%). LRT was compatible with the other excipients after 6 months. Particle size did not significantly alter particularly at RT. The highest release rate was obtained with NE $(1.339 \pm 0.026 \mathrm{mcg} / \mathrm{ml} / \mathrm{h})$ followed by NLC $(1.007 \pm 0.011 \mathrm{mcg} / \mathrm{m} / \mathrm{h})$ and $S L N(0.821 \pm 0.012 \mathrm{mcg} / \mathrm{m} / \mathrm{h})$, indicating anomalous transport $(p<0.05)$. Penetration profiles of LRT through skin were statistically similar for SLN and NLC $(p>0.05)$. NE showed the highest penetration rate $\left(0.829 \pm 0.06 \mathrm{mcg} / \mathrm{cm}^{2} / \mathrm{h}\right)(p<0.05)$.

Conclusion: SLN and NLC of LRT are alternative formulations for immediate treatment of allergic skin reactions with prolonged drug delivery via reservoir action.
\end{abstract}

Keywords: Loratadine, Transdermal delivery, Controlled drug delivery, Solid Lipid nanoparticles, Nanostructured lipid carriers, Allergy

Tropical Journal of Pharmaceutical Research is indexed by Science Citation Index (SciSearch), Scopus, International Pharmaceutical Abstract, Chemical Abstracts, Embase, Index Copernicus, EBSCO, African Index Medicus, JournalSeek, Journal Citation Reports/Science Edition, Directory of Open Access Journals (DOAJ), African Journal Online, Bioline International, Open-J-Gate and Pharmacy Abstracts

\section{INTRODUCTION}

Histamine which is present as a tissue hormone, especially in the skin and lungs in human, is also found in the bee poison and the salivary secretion of biting insects [1,2]. When allergic skin reactions occur or immediately after being bitten by an insect, excess histamine is released causing itching and formation of skin weals and flares. Even though histamine is rapidly metabolised in the body, the effects caused by the subsequent release may last for a considerable period of time which severely affects the well-being of the person concerned. 
The second generation of $\mathrm{H} 1$ receptor blocking agents including loratadine (LRT), can be used for inhibiting histamine release from the basophilic granulocytes and the mastocytes where histamine is stored in tissues [3]. LRT displays various side effects including several allergic reactions including rash, hives, itching, difficulty in breathing, tightness in the chest, swelling of the mouth or face and dizziness during systemic administration by oral route. Thus, topical preparations of LRT for skin application would be advantageous for the management of skin reactions. However, LRT has a poor penetration through the skin. This limitation may be overcome by incorporation of LRT into colloidal drug carrier systems like solid lipid nanoparticles (SLN) and nanostructured lipid carriers (NLC) $[4,5]$.

SLN and NLC are advantageous colloidal drug carrier systems over their colloidal counterparts such as polymeric nanoparticles, liposomes and microemulsions [4]. SLN are produced using solid lipids including high-melting point glycerides or waxes, replacing liquid lipid used in emulsions. Thus, SLN introduce prolonged delivery of actives by reservoir action. However, SLN may have some limitations in drug loading capacity depending on the solubility of the drug in the solid lipid and/or drug loss during storage due to lipid crystallization to the stable $\beta$-modification and relatively high water content. NLC, the second generation of SLN are produced by using spatially incompatible lipids together. Incorporation of liquid lipid into the solid structure ensures to overcome possible limitations related to SLN. SLN and NLC are sophisticated colloidal drug carrier systems suitable for skin applications due to their various desirable effects on skin. Occlusive properties of SLN and NLC on skin provide increase in drug penetration particularly via skin moisturization. They can transfer drugs into deeper layers of the skin, provide reservoir action and sustained drug delivery. They are also well suited for use on damaged or inflammed skin since they are based on non-irritant and non-toxic lipids. Thus, topical preparations of SLN and NLC have been increasingly attracted by industry for years and they are introduced to the market of European Community. In the meantime, high pressure homogenization which can be employed avoiding use of organic solvents has an excellent reproducibility in the large scale production for SLN and NLC as a cost-effective technique.

The objective of this study was to incorporate LRT into SLN and NLC with the aim to achieve skin penetration of LRT and to provide reservoir action for the treatment of disorders or diseases of the skin caused by excessive histamine release.

\section{EXPERIMENTAL}

\section{Materials}

Loratadine (LRT), Miglyol ${ }^{\circledR}$ 812s (Sasol Germany $\mathrm{GmbH}$, Germany) and TegoCare ${ }^{\circledR} 450$ (Evonik Goldschmidt $\mathrm{GmbH}$, Germany) were thankfully provided by TEVA Pharmaceutical Works Private Ltd. (Hungary), Kale Kimya (Kocaeli, Turkey) and Elkasan Kimyevi Maddeler Pazarlama A.Ş. (Istanbul, Turkey), respectively. 1-hexadecanol was purchased from Fluka. All the other chemicals used were of analytical grade.

\section{Preparation of SLN and NLC}

High pressure homogenization method was employed for production of nanoparticles $[4,5]$. TegoCare ${ }^{\circledR} 450(2 \% \mathrm{w} / \mathrm{w})$ and 1-hexadecanol (15 $\% \mathrm{w} / \mathrm{w}$ ) were used as surfactant and solid lipid for production of placebo SLN (PSLN), respectively. Solid lipid was melted at $75{ }^{\circ} \mathrm{C}$. Aqueous surfactant solution at the same temperature was added to the melted lipid using an Ultra-Turrax T25 homogenizer (Jahnke und Kunkel GmbH, Germany) at 20,000 rpm for 1 min. The coarse emulsion was passed through an APV-Lab 2000 high pressure homogenizer (Poland) with two steps (50 bar and 1500 bar, respectively) for three cycles. In the case of placebo NLC (PNLC), Miglyol $^{\circledR} 812$ s as liquid lipid was added to the solid lipid to reduce the fraction of 1-hexadecanol (1:2 w/w, respectively), i.e. the total lipid content stayed unchanged. PNLC was produced in exactly the same manner under the same conditions. Preparations were poured into transparent silanized vials and capped tightly. Vials were stored at RT, 4 and 40 ${ }^{\circ} \mathrm{C}$ in the dark. In the case of drug loaded nanoparticles (SLN and NLC), $1 \%$ LRT (w/w) of the total formulation was added to the lipophilic phase to reduce its fraction.

Only liquid lipid (Miglyol ${ }^{\circledR}$ 812s) was used instead of solid lipid in PNE and NE produced by using the same technique and conditions for comparison.

\section{Entrapment efficiency and loading capacity of SLN and NLC}

EE (\%) and LC (\%) of nanoparticles were determined by analysing the concentration of free LRT in the dispersion medium $[6,7]$. Nanoparticle dispersion was diluted with water at a certain rate. It was centrifuged at $13000 \mathrm{rpm}$ (Heraeus Biofuge Primo R Centrifuge, Thermo 
Fisher Scientific, Germany) for 40 min. Proper amount of supernatant was taken and diluted to $10 \mathrm{ml}$ with a mixture of phosphate buffer (PBS, $\mathrm{pH}$ 7.4) and propylene glycol mixture (60:40). The solution was analysed by HPLC. EE and LC were calculated using Eqs 1 and 2,

$E E=\{(W i-W f) / W i\} 100$

$\mathrm{LC}=\{(\mathrm{Wi}-\mathrm{Wf}) / \mathrm{Wp}\} 100$

where $\mathrm{Wi}$ and $\mathrm{Wp}$ are the amounts of initial drug and lipid used for the production of the nanoparticles, respectively, while $\mathrm{Wf}$ is the amount of free drug detected in the aqueous supernatant.

\section{FTIR analysis}

Determination of interaction between LRT and the other ingredients of SLN and NLC was investigated by FTIR after 6 months [8]. Nanoparticle dispersion was diluted with water and centrifuged at $13000 \mathrm{rpm}$ for $40 \mathrm{~min}$. The residue was transferred into glass plates and was kept at $40{ }^{\circ} \mathrm{C}$ over night for elimination of aqueous phase of dispersions. The sample was used for FTIR analysis. Residues obtained from SLN and NLC, and pure LRT were separately scanned over a wave number range of 4000 to $650 \mathrm{~cm}^{-1}$ at a resolution of $4 \mathrm{~cm}^{-1}$ in the transmission mode in a Perkin Elmer 100 FTIR instrument (UK) equipped with Perkin Elmer Spectrum Version 6.0.2 Software.

\section{DSC analysis}

DSC analysis was employed on pure lipid, placebo and drug loaded nanoparticles in order to investigate crystallization behaviour of lipid and drug-lipid interaction. $25 \mu \mathrm{l}$ samples equal to 3-4 mg solid content were weighed into standard sealed aluminium pans of the apparatus (DSC 204 F1 Phoenix ${ }^{\otimes}$, Netzsch, Germany) and heated from $20{ }^{\circ} \mathrm{C}$ to $80{ }^{\circ} \mathrm{C}$ with a heating rate of $10 \mathrm{~K} / \mathrm{min}$ under a nitrogen flow rate of $20 \mathrm{ml} / \mathrm{min}$. Melting peaks and enthalpies were calculated using the DSC 204 F1 software. Crystalline state of solid lipid formulations was demonstrated by calculation of crystallinity indice $(\mathrm{Cl})$ using $\mathrm{Eq} 3$ [9],

$\mathrm{Cl}(\%)=\{\mathrm{MEs} /(\mathrm{MEp} \times \mathrm{Cd})\} 100$

where MEs = melting enthalpy of the sample $(\mathrm{J} / \mathrm{g}), \mathrm{MEp}=$ melting enthalpy of pure solid lipid $(\mathrm{J} / \mathrm{g})$, and $\mathrm{Cd}=$ concentration of the solid lipid $(\%)$.

\section{Particle size measurement}

Particle size distribution of placebo and drug loaded formulations were determined in water by using a laser diffractometer equipped with Hydro 2000MU wet sample dispersion unit (Malvern Mastersizer Instrument, UK). Samples were diluted before the measurements $(n=3)$. Measurement medium was water with a refractive index of 1.33. Particle size of formulations were analysed as volumetric distribution (D10, D50 and D90).

\section{In vitro drug release}

Dialysis bag diffusion technique was used for determination of In vitro LRT release from SLN, NLC and NE under sink condition [7]. A sample $(1 \mathrm{~g})$ was placed in the regenerated cellulose dialysis bag (Mw cut-off at $3.500 \mathrm{Da}$, Spectra/Por ${ }^{\circledR}$ dialysis membrane, U.S.A) and both ends of the bag were tightly closed. The bag was immersed into a flask containing $200 \mathrm{ml}$ dissolution medium (PBS/propylene glycol, 60:40) that was placed in an agitating water bath. We used $40 \%$ propylene glycol in the receptor phase since previous studies used receptor phases containing up to $50 \%$ propylene glycol for in vitro release and ex vivo penetration experiments (10-12). The system was maintained at $37 \pm 0.5^{\circ} \mathrm{C}$ and agitated at $60 \mathrm{rpm}$. Samples $(1 \mathrm{ml}$ each) were collected at predetermined time intervals for $48 \mathrm{~h}$. Cumulative amounts of drug in the sample were detected by HPLC after proper dilution. This study was replicated 6 times for each formulations. The mean cumulative amount of LRT in dissolution medium was plotted as a function of time.

Release profiles were evaluated using different models in order to determine one that best describes release mechanism of drug: zero order, which describes the release rate independent of drug concentration; first order, which describes the release rate dependent of concentration gradient; Higuchi, which is based on the Fick's law of diffusion; KorsemeyerPeppas which indicates the Fickian diffusion and non-Fickian diffusion, where $\mathrm{n}$ is the release exponent, indicative of mechanism of drug release (Eqs 4 - 7) [8].

$$
\begin{aligned}
& \text { Zero order: } \mathrm{Q}_{\mathrm{t}}=\mathrm{Q}_{0}+\mathrm{k}_{0 \mathrm{t}} \ldots \ldots \ldots \ldots \ldots \ldots \text { (4) } \\
& \text { First order: } \\
& \mathrm{Q}_{\mathrm{t}}=\mathrm{Q}_{\infty}\left(1-\mathrm{e}^{-\mathrm{k}}{ }_{1}^{\mathrm{t}}\right) \text {..... } \\
& \text { Higuchi square-root: } \\
& \mathrm{Q}_{\mathrm{t}}=\mathrm{Q}_{0}+\mathrm{k}_{\mathrm{H}} \mathrm{t}^{1 / 2} \text {. } \\
& \text { Korsmeyer-Peppas: } \\
& \log \left[Q_{t} / Q_{\infty}\right]=\log k+\text { logt }
\end{aligned}
$$

where $Q_{t}$ and $Q_{0}=$ amounts of drug released at time, $\mathrm{t}$, and in the release medium at $\mathrm{t}=0$, respectively, $k_{0}, k_{1}$ and $k_{H}=$ release constants of the zero order, the first order and Higuchi 
square-root models, respectively, $Q_{\mathbb{t}} / Q_{\infty}=$ fractional release of drug, $k$ and $n=$ kinetic constant and diffusional release exponent indicative of the release mechanism.

\section{Penetration through excised rat skin}

Full thickness abdominal skins of Wistar albino rats $(150-200 \mathrm{~g})$ were obtained after rats were sacrificed [13]. Skins were depilated and dermal surfaces were carefully cleaned to remove subcutaneous tissues without damaging the epidermal surface. Underlying fatty tissue was removed by blunt dissection. The experimental protocol was approved by the Local Ethical Committee of Animal Experiments in Experimental Medical Research Institute of Istanbul University (2011/157) following the Council of Europe [14].

The formulation (1 g) was applied to the donor compartment on the surface of rat skins between two halves of Franz-type diffusion cell. Receptor phase was $33.2 \mathrm{ml}$ PBS/propylene glycol (60:40) at $37 \pm 1{ }^{\circ} \mathrm{C}[11,12]$. Amount of LRT penetrated through skin was determined from the samples collected at predetermined time intervals for $48 \mathrm{~h}$ by HPLC $(n=3)$. The steady state flux of LRT $\left(\mu \mathrm{g} / \mathrm{cm}^{2} / \mathrm{h}\right)$ was calculated from the slope of the linear portion of the plot of the cumulative amount permeated versus time.

\section{Analytical quantification of LRT by HPLC}

Quantification of LRT was made using a Shimadzu LC-2010HT HPLC apparatus (Japan) at RT. Data acquisition was performed with the software of Shimadzu LC-2010HT. Separations were provided by using a Phenomenex $\mathrm{C}_{18^{-}}$ column $(250 \mathrm{~mm} \times 4.6 \mathrm{~mm}$ i.d., $5 \mu \mathrm{m}$ particle size). A mobile phase consisting of acetonitrile and water (80:20) was used at a flow rate of 0.8 $\mathrm{ml} / \mathrm{min}$. Injection volume was $20 \mu \mathrm{L}$. Calibration curve was gained from 6 solutions of LRT in PBS/propylene glycol (60:40) at $0.1-1.0 \mu \mathrm{g} / \mathrm{ml}$ concentration range by detection at $200 \mathrm{~nm}$. The retention time of LRT was $\sim 2$ min.

\section{Statistical analysis}

Data obtained from studies on In vitro drug release and drug penetration through excised rat skin were evaluated using GraphPad Prism software and one-way ANOVA to determine differences between profiles. $P<0.05$ was set as the level of significance.

\section{RESULTS}

\section{LRT calibration}

The calibration curve was linear over the concentration range of $0.5-8.0 \mu \mathrm{g} / \mathrm{ml}\{\mathrm{y}=4.602$ e-06x $\left.+62.378\left(r^{2}=0.9989\right)\right\}$ by HPLC. The concentration of LRT in each samples were calculated from the corresponding regression equations.

\section{EE and LC of SLN and NLC}

EE of SLN and NLC was found as $90.67 \pm 0.48$ and $93.27 \pm 0.44 \%$ when LC values were obtained as $6.06 \pm 0.03$ and $6.22 \pm 0.03 \%$, respectively.

\section{FTIR spectra}

Pure LRT spectra showed very intense several characteristic IR absorption bands [8]. Their assignments of LRT were as follows: 1700.93 $\mathrm{cm}^{-1}(\mathrm{C}=\mathrm{O}$ stretching band $), 1643.37 \mathrm{~cm}^{-1} \quad(\mathrm{C}=\mathrm{N}$ stretching band), $1560.15 \mathrm{~cm}^{-1}-1434.13 \mathrm{~cm}^{-1}$ $(\mathrm{C}=\mathrm{C}$ stretching vibrations of benzene ring), $1385.05 \mathrm{~cm}^{-1}$ (N-C stretching band), 1222.30 $\mathrm{cm}^{-1}$ and $996.60 \mathrm{~cm}^{-1}$ (C-O stretching bands), $862.83 \mathrm{~cm}^{-1}$ (=C-H stretching band), 762.87 $\mathrm{cm}^{-1}$ ( $\mathrm{C}-\mathrm{Cl}$ stretching band). These findings have also been reported in various studies by different research groups $[15,16]$.

It was observed that $\mathrm{C}=\mathrm{O}$ stretching bands caused by aliphatic ester structure of triglycerides displayed sharp peaks at 1768.71 and $1722.16 \mathrm{~cm}^{-1}$ for SLN and NLC, respectively. $\mathrm{C}-\mathrm{H}$ and $\mathrm{O}-\mathrm{H}$ stretching bands of triglycerydes were detected at $3295.28 \mathrm{~cm}^{-1}-2835.43 \mathrm{~cm}^{-1}$. The same peaks were detected at the same wave number range after 6 months of storage of SLN and NLC at RT, 4 and $40^{\circ} \mathrm{C}$.

\section{DSC thermograms}

Heating of the bulk lipid caused thermal transitions. DSC thermogram of 1-hexadecanol showed sharp endothermic peak at $55.57{ }^{\circ} \mathrm{C}$ corresponding to the melting of $\beta$ polymorphic forms of the crystal lipid (Table 1). The onset value and the theoretical melting enthalpy of the solid lipid was determined as $49.47{ }^{\circ} \mathrm{C}$ and $235.76 \mathrm{~J} / \mathrm{g}$. According to data obtained from DSC experiments on formulations after their preparation, melting points of nanoparticles were detected to decrease to lower temperatures with broader peaks compared to bulk lipid by giving variable enthalpy values indicating several thermal transitions as well. Decrease in melting points of PSLN and SLN formulations was 
Table 1: DSC parameters of bulk lipid, placebo and drug loaded nanoparticles after 6 months of storage at room temperature (RT), 4 and $40^{\circ} \mathrm{C}$

\begin{tabular}{lcccc}
\hline Formulation & Storage temp $\left({ }^{\circ} \mathbf{C}\right)$ & Enthalpy $\mathbf{\Delta} \mathbf{H}(\mathbf{J} / \mathbf{g})$ & Melting point $\left({ }^{\circ} \mathbf{C}\right)$ & $\mathbf{C l}(\%)$ \\
\hline Bulk 1-hexadecanol & RT & 235.76 & 55.57 & 100 \\
PSLN & RT & 19.96 & 51.78 & 56.44 \\
& 4 & 20.67 & 51.83 & 58.45 \\
& 40 & 17.51 & 50.65 & 49.51 \\
SLN & RT & 22.79 & 50.47 & 64.44 \\
& 4 & 23.42 & 50.22 & 66.23 \\
& 40 & 19.96 & 50.51 & 56.44 \\
PNLC & RT & 14.39 & 48.24 & 40.69 \\
& 4 & 14.82 & 47.93 & 41.91 \\
& 40 & 13.08 & 47.86 & 36.99 \\
NLC & RT & 15.71 & 46.79 & 44.42 \\
& 4 & 15.91 & 47.53 & 44.99 \\
& 40 & 12.43 & 46.16 & 35.15 \\
\hline
\end{tabular}

$R T=$ room temperature, $\mathrm{Cl}=$ crystallinity indice

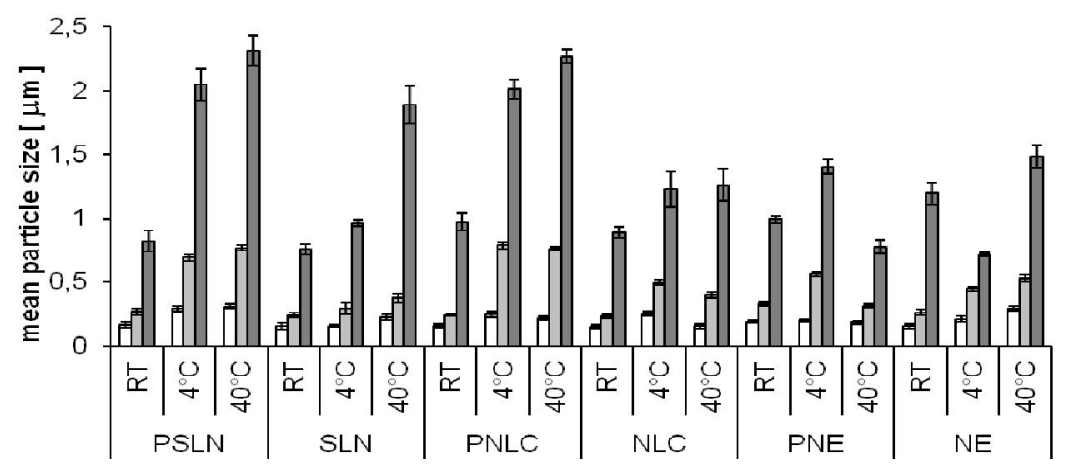

Figure 1: D10 (口), D50 ( $\square$ ) and D90 ( $₫$ ) values of placebo and drug loaded nanoparticles and NEs after 6 months of storage. Note: Laser diffraction (LD) data = volume distribution

Table 2: Determination coefficients for drug release from SLN, NLC and NE with diffusion exponents of release profiles for Korsmeyer Peppas model

\begin{tabular}{ccccc}
\hline Formulations & Zero order & First order & Higuchi & $\begin{array}{c}\text { Korsmeyer-Peppas } \\
(\boldsymbol{n})\end{array}$ \\
\hline SLN & 0.9044 & 0.6977 & 0.9773 & $\mathbf{0 . 9 8 4 8}(0.5281)$ \\
NLC & 0.9345 & 0.7742 & 0.9904 & $\mathbf{0 . 9 9 3 2}(0.4824)$ \\
NE & 0.8828 & 0.7225 & 0.9850 & $\mathbf{0 . 9 9 1 2}(0.4604)$ \\
\hline
\end{tabular}

$R^{2}=$ correlation coefficient; $n=$ diffusion exponent of release profile (slope). Best fits were bolded.

detected as $5{ }^{\circ} \mathrm{C}$ when incorporation of liquid lipid causes more decrease in case of PNLC and NLC. Table 1 lists various parameters obtained from samples after 6 months of storage at RT, 4 and $40{ }^{\circ} \mathrm{C}$.

\section{Particle size}

D50 values of placebo formulations (PSLN, PNLC and PNE) obtained from LD were in 0.229 $-0.258 \mu \mathrm{m}$ size range in their production week. LRT incorporation into formulations did not significantly alter their particle size. SLN displayed the highest D50 value $(0.252 \pm 0.008$ $\mu \mathrm{m})$ followed by NLC $(0.248 \pm 0.010 \mu \mathrm{m})$ and NE $(0.222 \pm 0.002 \mu \mathrm{m})$. Figure 1 presents data obtained from placebo and drug loaded nanoparticles and NE formulation after 6 months of storage at different temperatures.

\section{In vitro drug release}

LRT release was the lowest with SLN followed by NLC and NE, respectively as can be seen in 
Figure $2(p<0.05)$. Release rate of LRT from the different formulations were $0.821 \pm 0.018,1.007$ \pm 0.011 and $1.339 \pm 0.012 \mathrm{mcg} / \mathrm{ml} / \mathrm{h}$.

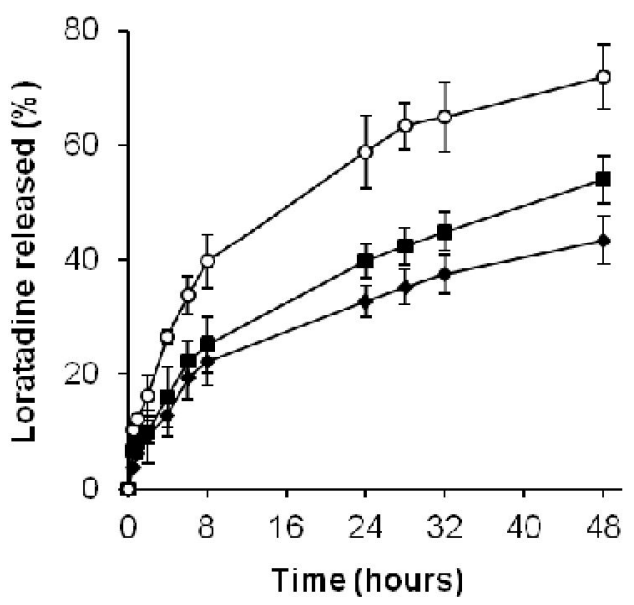

Figure 2: Release profiles of LRT from SLN ( $\bullet$, NLC (匹) and NE (o)

Korsmeyer-Peppas was shown to be the best fitting model for all formulations (Table 2). A combined pattern of diffusion and erosion release mechanism (anomalous non-Fickian transport, $0.45 \leq \mathrm{n}<0.89$ ) was found for SLN and NLC which shows the ability of the system for controlled drug release. NE was also displayed the same release mechanism closer to $0.45 \mathrm{n}$ value, which indicates predominantly diffusional drug release.

\section{Penetration through excised rat skin}

The highest penetration for LRT through skin occured with NE followed by NLC and SLN $(p<$ 0.05 ) (Figure 3). However, insignificant difference was obtained between LRT penetration profiles of SLN and NLC. Drug penetration reached to a steady state flux between 8th and 24th hours. In this period, penetration rates were found as $0.707 \pm 0.008,0.725 \pm 0.010$ and $1.123 \pm 0.014$ $\mathrm{mcg} / \mathrm{cm}^{2} / \mathrm{h}$ for SLN, NLC and NE, respectively.

\section{DISCUSSION}

Drug payload of SLN and NLC was high as it was desired for a colloidal drug carrier system. Addition of liquid lipid to the lipophilic phase resulted in a slight increase in drug payload as reported earlier $[7,8]$. This was attributed to the higher solubility of LRT in liquid lipid compared to the solid lipid. Interaction between LRT and excipients were physical. FTIR spectra of samples exhibited peaks of LRT and triglycerides in the finger print area indicating chemical compatibility during 6 months of storage at RT, 4 and $40{ }^{\circ} \mathrm{C}$.

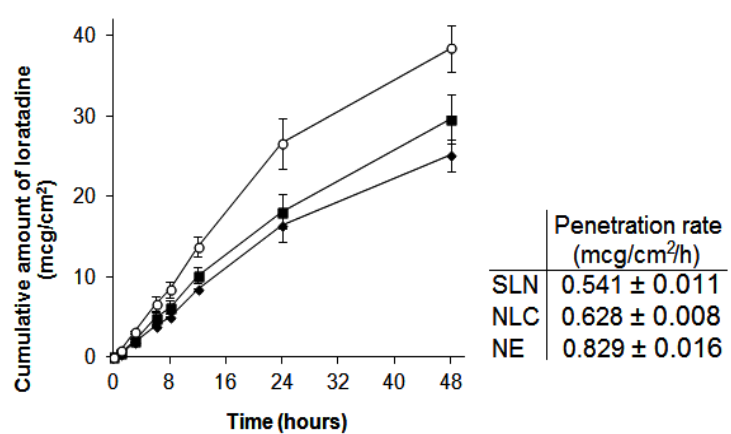

Figure 3: LRT penetration through full thickness abdominal skins of Wistar Albino Rats from SLN (»), NLC ( $\mathbf{})$ and NE (o)

DSC gave information on thermal behaviours of nanoparticles. Reduction in Cl of PNLC and NLC compared to PSLN and SLN could be attributed to the crystal order in PNLC and NLC greatly disturbed due to the oil inside the particles. Liquid lipid prevented crystallization of lipid to $\beta$ form [17]. It was thought that this situation strictly affected drug accommodation in nanoparticles. Uncompleted crystallization or imperfect crystal order provided accommodation of the drug in molecular form. Storage temperature was also seen to affect the degree of crystallinity of nanoparticles. This difference was particularly obvious between 4 and $40{ }^{\circ} \mathrm{C}$.

RT was the most suitable storage condition for SLN and NLC since weak agglomeration resulted at 4 and $40{ }^{\circ} \mathrm{C}$. In this case, D50 values of SLN and NLC were lower than $0.800 \mu \mathrm{m}$ while LD profiles exhibited low microparticle content showing that SLN and NLC displayed good physical stability [18]. Nanometer size range of SLN and NLC can provide occlusive effect leading to an increase in skin hydration by their close contact with the stratum corneum $[5,19]$. Skin hydration can cause subsequent increase in skin penetration of a drug or transfer of it into deeper layers of skin.

Oil content in NLC caused enhancement of drug release rate from nanoparticles. Existence of liquid lipid in NLC spoiled formation of crystal structure in the matrix and makes drug liberation to a medium where the drug is readily soluble. In the case of NE, drug release was faster since its lipophilic phase was composed of liquid lipid. Release of LRT from SLN and NLC continued at the same rate up to the end of the 8th hour which was the last sampling time of the first day of the study. Difference and decrease in release rate were detected from the 8th hour. Biphasic pattern of release profiles can be explained by non-Fickian diffusion (anomalous transport) described by Korsmeyer-Peppas model. When nanoparticles were exposed to the release 
medium, drug close to the surface of nanoparticles was released by diffusion - within the first $4 \mathrm{~h}$ and erosion of nanoparticles appeared gradually. Subsequent release from the 8th hour continued in a linear manner $(r=$ 0.9867 and $r=0.9892$ for SLN and NLC, respectively).

Lipophilic phase composed of oil in NE led faster drug penetration rate through skin since it provided drug release convenient after close contact with Stratum corneum. Liberation of LRT from droplets of NE was occured more easily compared to the matrix structure of SLN and NLC as could be expected.

In a recent study, SLN and NLC were reported to exhibit a high adhesion to the stratum corneum enhancing the amount of drug which penetrated into the viable skin [19]. Lipid nanoparticles formulated in this study are expected to display an occlusive effect and reduce the transepidermal water loss. This will result in an enhancement in amount of drug penetrated into the skin layers.

\section{CONCLUSION}

SLN and NLC of LRT were prepared with good steric stabilization, high drug payload and crystal properties leading drug accommodation in nanoparticles during storage. SLN and NLC introduced colloidal carrier systems with desirable controlled release characteristics for LRT. It can be concluded that SLN and NLC can be used for immediate treatment of allergic skin reactions and then prolonged drug delivery via reservoir action in order to prevent excess histamin release. NE of LRT, which was produced for comparison in order to characterize SLN and NLC was also found a physically stable formulation at different storage temperatures after 6 months. It displayed the highest penetration rate through skin. Thus, it was found to have a high potential for the treatment of allergic skin reactions.

\section{CONFLICT OF INTEREST}

The authors declare that they have no conflict of interest to disclose.

\section{ACKNOWLEDGEMENT}

The authors would like to thank Biofarma Pharmaceutical Industry Co Inc, Istanbul and Argis Pharmaceutical Research and Development, Ankara for their contribution.

\section{REFERENCES}

1. Horsmanheimo L, Harvima IT, Harvima RJ, BrummerKorvenkontio H, François G, Reunala T. Histamine and leukotriene $C 4$ release in cutaneous mosquitobite reactions. J Allergy Clin Immunol 1996; 98(2): 408-411.

2. Kiningham KK. Loratadine. xPharm: The Comprehensive Pharmacology Reference, 2007, pp 1-4.

3. Hadzijusufovic E, Peter B, Gleixner KV, Schuch K, PickI WF, Thaiwong T, Yuzbasiyan-Gurkan V, Mirkina I, Willmann $M$, Valent $P$. H1-receptor antagonists terfenadine and loratadine inhibit spontaneous growth of neoplastic mast cells. Exp Hematol 2010; 38(10): 896-907.

4. Üner M. Preparation, characterization and physicochemical properties of solid lipid nanoparticles (SLN) and nanostructured lipid carriers (NLC): their benefits as colloidal drug carrier systems. Pharmazie 2006; 61(5): 375-386.

5. Wissing SA, Lippacher A, Muller RH (2001) Investigations on the occlusive properties of solid lipid nanoparticles (SLN). J Cosmet Sci 52(5): 313324.

6. Kuo Y-C, Chen $\mathrm{H}-\mathrm{H}$. Entrapment and release of saquinavir using novel cationic solid lipid nanoparticles. Int J Pharm 2009; 365(1-2): 206-213.

7. Ghadiri M, Fatemi S, Vatanara A, Doroud D, Najafabadi AR, Darabi M, Rahimi AA. Loading hydrophilic drug in solid lipid media as nanoparticles: Statistical modeling of entrapment efficiency and particle size. Int J Pharm 2012; 424(1-2): 128-137.

8. Üner M, Karaman EF. Preliminary studies on solid lipid microparticles of loratadine for the treatment of allergic reactions via the nasal route. Trop $J$ Pharm Res 2013; 12(3): 287-293.

9. Siekmann B, Westesen $K$. Thermoanalysis of the recrystallization process of melt-homogenized glyceride nanoparticles. Colloid Surface B 1994; 3(3): 159-175.

10. Ren X, Svirskis D, Alany RG, Zargar-Shoshtari S, Wu Z. In-situ phase transition from microemulsion to liquid crystal with the potential of prolonged parenteral drug delivery. Int J Pharm 2012; 431(1-2): 130-137.

11. Hosmer J, Reed R, Bentley MVLB, Nornoo A, Lopes LB. Microemulsions containing medium-chain glycerides as transdermal delivery systems for hydrophilic and hydrophobic drugs. AAPS Pharm Sci Tech. 2009; 10(2): 589-596.

12. Biruss $B$, Valenta $C$. The advantage of polymer addition to a non-ionic oil in water microemulsion for the dermal delivery of progesterone. Int J Pharm. 2008; 349(1-2): 269-73.

13. Yener G, Üner M, Gönüllü Ü, Yıldırım S, Kılıç $P$, Sağlık Aslan S, Barla A. Design of meloxicam and lornoxicam transdermal patches: Preparation, physical characterization, ex vivo and in vivo studies. Chem Pharm Bull 2010; 58(11): 1466-1473.

14. European Convention for the Protection of Vertebrate Animals Used for Experimental and Other Scientific Purposes. Strasbourg, 1986.

15. Lin S-Y, Hsu C-H, Sheu M-T. Curve-fitting FTIR studies of loratadine/hydroxypropyl- $\beta$-cyclodextrin inclusion complex induced by co-grinding process. J Pharm Biomed Anal 2010; 53(3): 799-803.

16. Nayak RK, Manjunath B, Narayana Swamy VB, Senthil A, Thakkar HK, Kumar DM, Mahalaxmi R. Design and evaluation of sustained release floating tablets of loratadine. Asian J Biochem Pharm Res 2011; 1(3): 105-124.

17. How CW, Abdullah $R$, Abbasalipourkabir $R$. Physicochemical properties of nanostructured lipid carriers as colloidal carrier system stabilized with 
polysorbate 20 and polysorbate 80 . African Biotechnol 2011; 10(9): 1684-1689.

18. Heurtault B, Saulnier P, Pech B, Proust JE, Benoit JP.

Physico-chemical stability of colloidal lipid particles. Biomaterials 2003; 24(23): 4283-4300.
19. Li B, Ge Z-Q. Nanostructured lipid carriers improve skin permeation and chemical stability of idebenone. AAPS Pharm Sci Tech 2012; 13(1): 276-283. 